\title{
RESPONSE OF DIFFERENT STORAGE CONTAINERS IN RICE GENOTYPES DURING STORAGE
}

\author{
Dr. Sanjoy Kumar Bordolui \\ Assistant Professor \\ Department of Seed Science and Technology \\ Bidhan Chandra Krishi Viswavidyalaya \\ Mohanpur - 741252, Nadia, West Bengal, India \\ E-mail: sanjoy_bordolui@rediffmail.com \\ Nagapuri Sachin Kumar \\ PG Student \\ Department of Seed Science and Technology \\ Bidhan Chandra Krishi Viswavidyalaya \\ Mohanpur - 741252, Nadia, West Bengal, India \\ E-mail: sachinnagapuri@gmail.com \\ Dr. Kanu Murmu \\ Assistant Professor \\ Department of Agronomy \\ Bidhan Chandra Krishi Viswavidyalaya \\ Mohanpur - 741252, Nadia, West Bengal, India \\ E-mail: kanumurmu@gmail.com
}

\begin{abstract}
Rice is the staple food of more than half the world's population and is being cultivated in Asia since ancient time's generation after generation. Good quality seeds are the seeds of the green revolution, it has been assertively and emphatically shown that 15-20\% increased yield could be achieved by the use of good quality seeds alone. Good quality seed is one of the most important requirements for production and supply to achieve higher production and productivity in any crop. The seed quality is depended on various factors Viz. genetic, edaphic, environmental, biotic, etc. Good quality seed production depends on complex conditions evoking the most favorable interactions between the genetic makeup of the seed and the environment, under which it is produced, harvested, processed, and stored. Seeds undergo deterioration at various levels during storage resulting in a decline in vigor and viability. With the above considerations, the present investigation has been undertaken to observe the potentials of ten rice genotypes and observed its seed quality after harvesting in 2016-2017 at Seed Testing Laboratory, Department of Seed Science and Technology, Bidhan Chandra Krishi Viswavidyalaya, Mohanpur, Nadia, West Bengal, India. Just after harvesting $G_{2}$ (Gobindabhog) and $G_{9}$ (IR-36) showed highest germination percentage (97.66) and $G_{9}(I R-36)$ showed highest Vigor index (3204.13). But most of the varieties germination and vigor were nearer to $G_{2} \& G_{9}$. Among the containers with seed moisture content aluminum foil packet with seed moisture content, 8\% is the best storage
\end{abstract}


container for rice among the other containers as up to one-year storage vigor index was observed highest in that conditions.

Keywords: Containers, Germination, Rice, Storage, Vigor.

\section{INTRODUCTION}

Rice (Oryza sativa L.) is the major staple food and is being cultivated in Asia since ancient time's generation after generation. The landraces and wild species, the genetic resources of rice also include the natural hybrids and a range of different genetic stocks comprising commercial and absolute varieties (Biswas et al., 2020). Seeds undergo deterioration at various levels during storage resulting in decline in a vigor and viability (Bordolui et al., 2015). The loss of viability during storage due to atmospheric factors from the time of maturity is a common phenomenon in all crop seeds until the next planting season (Ramanadane et al., 2005; Chandra et al., 2006). The deterioration leading to a loss in viability of seed may affect the yield of the crop in two ways: firstly, decrease in germination may lead to a suboptimal population of plants per unit area, secondly poor performance by the surviving plants (Roberts, 1972). Maintenance of viability, vigor, and storability of seeds is a problem in tropical regions where high temperature and high relative humidity accelerate seed deterioration which ultimately results in non-viability (Bordolui et al., 2018). Over the years, researchers have, involved themselves to develop more suitable techniques to keep the seed healthy, viable, and vigorous during storage. With this available background information, the present investigation is proposed to evaluate the morphology and quality of produced seeds.

\section{MATERIALS AND METHODS}

The panicles of ten rice genotypes Giz, Kailash $\left(\mathrm{G}_{1}\right)$, Gobindabhog $\left(\mathrm{G}_{2}\right)$, Kalma $\left(\mathrm{G}_{3}\right)$, Dudheswar $\left(\mathrm{G}_{4}\right)$, Malabati $\left(\mathrm{G}_{5}\right)$, Kanakchur $\left(\mathrm{G}_{6}\right)$, Kalojira $\left(\mathrm{G}_{7}\right)$, Radhatilak $\left(\mathrm{G}_{8}\right)$, IR-36 $\left(\mathrm{G}_{9}\right)$ and Pusa-1509 $\left(\mathrm{G}_{10}\right)$ were collected from the trial plot of Gontra village, Nadia, West Bengal. The laboratory experiment was carried out in seed testing laboratory, Department of Seed Science and Technology, Bidhan Chandra Krishi Viswavidyalaya, Mohanpur, Nadia, West Bengal, India, during 2016-2017. Immediately after harvesting, seeds were properly sun-dried to its safe moisture content of rice seed collected from control plot of individual genotype for each replication were kept in four different packaging materials and one condition Viz., Different treatments were earthen pot with seed moisture content $10 \%-\mathrm{T}_{1}$, cloth bag with seed moisture content $10 \%-\mathrm{T}_{2}$, aluminum foil packet with seed moisture content $10 \%-\mathrm{T}_{3}$, aluminum foil packet with seed moisture content $8 \%-\mathrm{T}_{4}$ and polythene packet with seed moisture content $10 \%$ $\mathrm{T}_{5}$. Different seed quality parameters such as root length $(\mathrm{cm})$, shoot length $(\mathrm{cm})$, seedling length $(\mathrm{cm})$, germination percentage, and vigor index were recorded/determined at the pre-storage condition as well as at every three months interval up to one year of storage (Bordolui et al., 2006). The seedling vigor index was computed using the formula suggested by Abdul Baki and Anderson (1973) and data were analyzed by employing CRD through SASSA, DMRT.

\section{RESULTS AND DISCUSSION}

Among the genotypes, significant variation was recorded for test weight, seedling root length, fresh weight, and dry weight only. Among the genotypes mean value of test weight varied from $11.84 \mathrm{~g}$ for $\mathrm{G}_{2}$ to 22.27 for $\mathrm{G}_{1}$, and the deviation from the mean value was recorded from1.00 to 1.53. Constitution of different groups was: group A was constituted with single 
genotypes $\mathrm{G}_{10}$, B with two constituents $\mathrm{G}_{1}$ \& $\mathrm{G}_{3}$, group $\mathrm{C}$ with $\mathrm{G}_{4}$ \& $\mathrm{G}_{9}$ and remaining genotypes constituted group $\mathrm{D}$. Average seedling shoot length varied from $10.81 \mathrm{~cm} \mathrm{G}_{1}$ to 14.76 $\mathrm{cm} \mathrm{G}_{7}$. As the genotypes displayed non-significant variation in performance for this character, they formed a single group $\mathrm{A}$. For $\mathrm{G}_{2}$, while $\mathrm{G}_{9}$ exhibited the highest root length $(19.94 \mathrm{~cm})$ and it was the lowest $(11.24 \mathrm{~cm})$ for $\mathrm{G}_{2}$, while the deviation from the mean value varied from 0.57 to 4.10. The genotypes formed different groups as $G_{1}$ was grouped under $D C, G_{2}$ was under $D, G_{3}$ under $A B$ group, BAC was formed with $\mathrm{G}_{4}, \mathrm{G}_{5}$ and $\mathrm{G}_{7}$, while $\mathrm{G}_{6}$ and $\mathrm{G}_{8}$ were grouped under $B C D$, and $G_{10} \& G_{9}$ were grouped under $A B$ and $A$ respectively. Seedling fresh weighs highest $(1.60 \mathrm{~g})$ for $\mathrm{G}_{9}$ and it was lowest $(1.25 \mathrm{~g})$ for $\mathrm{G}_{2}$. As the genotypes displayed significant variation performance for this character, they formed different groups: $G_{1}$ was grouped under the $H$ group, $G_{2}$ was grouped under $J$, both $G_{3}$ and $G_{9}$ were grouped under $A, G_{4}$ was grouped under $G, G_{5}$ was grouped under $B, \mathrm{G}_{6}$ was grouped under $\mathrm{E}, \mathrm{G}_{7}$ was grouped under $\mathrm{F}, \mathrm{G}_{8}$ was grouped under $\mathrm{I}$, and $\mathrm{G}_{10}$ was grouped under $\mathrm{C}$. While considering seedling dry weight the mean value was highest $(0.95 \mathrm{~g})$ for $\mathrm{G}_{4}$ and lowest $(0.52 \mathrm{~g})$ for $\mathrm{G}_{8}$. The genotypes constituted different groups with varying constituents: the genotype $\mathrm{G}_{1}$ was grouped under $F, \mathrm{G}_{2}$ was under $\mathrm{G}, \mathrm{G}_{5}$ was under $\mathrm{B}, \mathrm{G}_{4}$ was under $\mathrm{A}, \mathrm{G}_{3}$ and $\mathrm{G}_{9}$ were under $\mathrm{C}, \mathrm{G}_{7}$ was under $\mathrm{E}, \mathrm{G}_{6}$ was under $\mathrm{D}, \mathrm{G}_{8}$ was under I group, and $\mathrm{G}_{10}$ was grouped under $\mathrm{H}$ group. Maximum germination $(97.66 \%)$ was recorded for both $\mathrm{G}_{2}$ and $\mathrm{G}_{9}$ though non-significant and it was minimum $(96.66 \%)$ for $\mathrm{G}_{5}, \mathrm{G}_{7}$, and $\mathrm{G}_{3}$, while the deviation from the mean value varied from 0.52 to 1.15 . The genotypes failed to constitute different groups due to their highest magnitude for vigor index (3204.13) was recorded for $\mathrm{G}_{9}$ and it was lowest for $\mathrm{G}_{10}$ (2009.17). Though non-significant variation among genotypes was noted for this character, genotype $G_{1}$ was grouped under $B, G_{2}$ was grouped under $C, G_{3}$ and $G_{9}$ were under $A$ group, and $\mathrm{G}_{10}$ was grouped under $\mathrm{BA}, \mathrm{G}_{8}$ was grouped under BAC.

So, harvested fresh seeds of $\mathrm{G}_{2}$ (Gobindabhog) \& $\mathrm{G}_{9}$ (IR-36) showed the highest germination percentage and that of $\mathrm{G}_{9}$ (IR-36) showed the highest Vigor index. There is no effect of different storage containers in fresh harvest seed.

Table 1. Mean value with standard deviation for test weight, seedling root-shoot length and fresh, dry weight, germination (\%) and vigor index of the genotypes at harvest fresh condition.

\begin{tabular}{|c|c|c|c|c|c|c|c|}
\hline Genotypes & $\begin{array}{c}\text { Test weight } \\
(\mathrm{g}) \\
\text { Mean } \pm \text { SD }\end{array}$ & $\begin{array}{l}\text { Shoot length } \\
(\mathrm{cm}) \\
\text { Mean } \pm \text { SD }\end{array}$ & $\begin{array}{l}\text { Root length } \\
(\mathrm{cm}) \\
\text { Mean } \pm \text { SD }\end{array}$ & $\begin{array}{c}\text { Fresh } \\
\text { weight }(\mathrm{g}) \\
\text { Mean } \pm \text { SD }\end{array}$ & $\begin{array}{c}\text { Dry } \\
\text { Weight (g) } \\
\text { Mean } \pm \text { SD }\end{array}$ & $\begin{array}{c}\text { Germination } \% \\
\text { Mean } \pm \text { SD }\end{array}$ & G.I. Mean \pm SD \\
\hline $\mathrm{G}_{1}$ & $22.27^{\mathrm{B}} \pm 1.53$ & $10.81^{\mathrm{A}} \pm 3.05$ & $13.0^{\mathrm{DC}} \pm 0.57$ & $1.29^{\mathrm{H}} \pm 0.00$ & $0.63^{\mathrm{F}} \pm 0.00$ & $97.00^{\mathrm{A}} \pm 1.00$ & $2312.86^{\mathrm{B}} \pm 335.36$ \\
\hline $\mathrm{G}_{2}$ & $11.84^{\mathrm{D}} \pm 1.53$ & $11.44^{\mathrm{A}} \pm 1.52$ & $11.24^{\mathrm{D}} \pm 0.57$ & $1.25^{\mathrm{J}} \pm 00.0$ & $0.63^{\mathrm{G}} \pm 0.00$ & $97.66^{\mathrm{A}} \pm 0.57$ & $2216.49^{C} \pm 152.66$ \\
\hline $\mathrm{G}_{3}$ & .53 & 2.51 & $18.82^{\mathrm{AB}} \pm 0.57$ & $1.45^{\mathrm{A}_{ \pm}}$ & 0.91 & 96.6 & 3137.9 \\
\hline $\mathrm{G}_{4}$ & \pm 1.53 & $13.78^{\mathrm{A}} \pm 2.51$ & $16.98^{\mathrm{BAC}_{ \pm}}+1.52$ & $1.30^{\mathrm{G}} \pm 0.00$ & $0.95^{\mathrm{A}} \pm 00.0$ & $97.00^{\mathrm{A}} \pm 1.00$ & $2986.70^{\mathrm{BA}} \pm 397.35$ \\
\hline $\mathrm{G}_{5}$ & $13.89^{\mathrm{D}} \pm 1.53$ & $13.85^{\mathrm{A}} \pm 2.51$ & $17.18^{\mathrm{BAC}} \pm 2.30$ & $1.52^{\mathrm{B}} \pm 0.00$ & $0.92^{\mathrm{B}} \pm 0.00$ & $96.66^{\mathrm{A}} \pm 0.57$ & $3001.82^{\mathrm{BA}} \pm 463.24$ \\
\hline $\mathrm{G}_{6}$ & $13.51^{\mathrm{D}} \pm 1.53$ & $12.86^{\mathrm{A}} \pm 2.51$ & $15.29^{\mathrm{BCD}} \pm 4.10$ & $1.38^{\mathrm{E}} \pm 0.00$ & $0.85^{\mathrm{D}} \pm 0.00$ & $97.00^{\mathrm{A}} \pm 1.00$ & $2981.82^{\mathrm{BA}} \pm 575.41$ \\
\hline $\mathrm{G}_{7}$ & $13.47^{\mathrm{D}} \pm 1.53$ & $14.76^{\mathrm{A}} \pm 2.51$ & $16.81^{\mathrm{ABC}} \pm 3.21$ & $1.31^{\mathrm{F}} \pm 0.00$ & $0.72^{\mathrm{E}} \pm 0.00$ & $96.66^{\mathrm{A}} \pm 0.57$ & $3053.30^{\mathrm{BA}} \pm 541.44$ \\
\hline $\mathrm{G}_{8}$ & $12.74^{\mathrm{D}} \pm 1.53$ & $12.60^{\mathrm{A}} \pm 2.51$ & $15.32^{\mathrm{BCD}} \pm 4.58$ & $1.26^{\mathrm{I}} \pm 0.00$ & $0.52^{\mathrm{I}} \pm 0.00$ & $97.33^{\mathrm{A}} \pm 1.15$ & $2721.45^{\mathrm{BAC}} \pm 618.78$ \\
\hline $\mathrm{G}_{9}$ & $18.44^{\mathrm{C}} \pm 1.00$ & $12.85^{\mathrm{A}} \pm 2.51$ & $19.94^{\mathrm{A}} \pm 0.57$ & $1.60^{\mathrm{A}} \pm 0.00$ & $0.91^{\mathrm{C}} \pm 0.00$ & $97.66^{\mathrm{A}} \pm 0.57$ & $3204.13^{\mathrm{A}} \pm 271.25$ \\
\hline $\mathrm{G}_{10}$ & $13.49^{\mathrm{A}} \pm 1.53$ & $13.24^{\mathrm{A}} \pm 2$ & $17.65^{\mathrm{AB}} \pm 3.21$ & $1.50^{\mathrm{C}} \pm 0.00$ & $0.62^{\mathrm{H}} \pm 0.00$ & $97.33^{\mathrm{A}} \pm 1.15$ & $2009.17^{\mathrm{BA}} \pm 502.70$ \\
\hline & 0.331 & & & 0.01 & 0.01 & 191 & 97.694 \\
\hline LSD (0.05) & $1.1262 *$ & NS & $1.4414^{*}$ & $\mathbf{0 . 0 2 7} *$ & $0.026 *$ & NS & NS \\
\hline
\end{tabular}

NB: Kailash- $\mathrm{G}_{1}$, Gobindabhog- $\mathrm{G}_{2}$, Kalma- $\mathrm{G}_{3}$, Dudheswar- $\mathrm{G}_{4}$, Malabati- $\mathrm{G}_{5}$, Kanakchur- $\mathrm{G}_{6}$, Kalojira- G7, Radhatilak- G8, IR36- $\mathrm{G}_{9}$ and Pusa-1509- $\mathrm{G}_{10}$. 
Table 2. Mean values for seedling shoot length, root length, fresh weight, dry weight, germination (\%), and vigor index of the genotypes stored in different containers at three months after storage

\begin{tabular}{|c|c|c|c|c|c|c|}
\hline $\begin{array}{l}\text { Genotypes with } \\
\text { treatments }\end{array}$ & $\begin{array}{l}\text { Shoot } \\
\text { length } \\
(\mathrm{cm})\end{array}$ & $\begin{array}{l}\text { Root } \\
\text { length } \\
(\mathrm{cm})\end{array}$ & $\begin{array}{c}\text { Fresh } \\
\text { weight }(\mathrm{g})\end{array}$ & $\begin{array}{c}\text { Dry } \\
\text { weight (g) }\end{array}$ & $\begin{array}{l}\text { Germination } \\
(\%)\end{array}$ & $\begin{array}{l}\text { Vigor } \\
\text { index }\end{array}$ \\
\hline $\mathrm{T}_{1} \mathrm{G}_{1}$ & 10.50 & 18.11 & 0.24 & 0.07 & 89.00 & 2548.62 \\
\hline $\mathrm{T}_{1} \mathrm{G}_{2}$ & 6.24 & 9.12 & 0.41 & 0.04 & 91.00 & 1395.95 \\
\hline $\mathrm{T}_{1} \mathrm{G}_{3}$ & 13.40 & 16.43 & 0.25 & 0.08 & 90.67 & 2705.20 \\
\hline $\mathrm{T}_{1} \mathrm{G}_{4}$ & 8.96 & 12.42 & 0.22 & 0.05 & 91.00 & 1949.28 \\
\hline $\mathrm{T}_{1} \mathrm{G}_{5}$ & 9.80 & 17.56 & 0.24 & 0.05 & 93.00 & 2545.28 \\
\hline $\mathrm{T}_{1} \mathrm{G}_{6}$ & 7.14 & 9.16 & 0.23 & 0.05 & 90.00 & 1468.76 \\
\hline $\mathrm{T}_{1} \mathrm{G}_{7}$ & 8.22 & 10.12 & 0.35 & 0.08 & 91.67 & 1683.45 \\
\hline $\mathrm{T}_{1} \mathrm{G}_{8}$ & 6.42 & 8.50 & 0.13 & 0.04 & 88.67 & 1320.96 \\
\hline $\mathrm{T}_{1} \mathrm{G}_{9}$ & 9.35 & 18.61 & 0.16 & 0.06 & 91.33 & 2579.19 \\
\hline $\mathrm{T}_{1} \mathrm{G}_{10}$ & 12.83 & 20.02 & 0.29 & 0.11 & 91.33 & 2995.17 \\
\hline $\mathrm{T}_{2} \mathrm{G}_{1}$ & 9.29 & 16.96 & 0.21 & 0.07 & 88.67 & 2323.13 \\
\hline $\mathrm{T}_{2} \mathrm{G}_{2}$ & 6.05 & 7.79 & 0.09 & 0.02 & 91.00 & 1257.41 \\
\hline $\mathrm{T}_{2} \mathrm{G}_{3}$ & 11.07 & 16.40 & 0.15 & 0.06 & 91.33 & 2512.09 \\
\hline $\mathrm{T}_{2} \mathrm{G}_{4}$ & 8.06 & 13.93 & 0.17 & 0.06 & 90.00 & 1979.61 \\
\hline $\mathrm{T}_{2} \mathrm{G}_{5}$ & 10.17 & 18.61 & 0.26 & 0.07 & 91.00 & 2616.23 \\
\hline $\mathrm{T}_{2} \mathrm{G}_{6}$ & 5.64 & 8.81 & 0.15 & 0.03 & 91.67 & 1326.49 \\
\hline $\mathrm{T}_{2} \mathrm{G}_{7}$ & 5.73 & 9.04 & 0.13 & 0.03 & 90.33 & 1334.85 \\
\hline $\mathrm{T}_{2} \mathrm{G}_{8}$ & 8.18 & 11.20 & 0.21 & 0.04 & 89.00 & 1725.63 \\
\hline $\mathrm{T}_{2} \mathrm{G}_{9}$ & 13.52 & 16.85 & 0.17 & 0.07 & 91.00 & 2764.77 \\
\hline $\mathrm{T}_{2} \mathrm{G}_{10}$ & 13.28 & 19.42 & 0.25 & 0.09 & 90.67 & 2962.96 \\
\hline $\mathrm{T}_{3} \mathrm{G}_{1}$ & 8.50 & 20.95 & 0.14 & 0.06 & 90.33 & 2661.55 \\
\hline $\mathrm{T}_{3} \mathrm{G}_{2}$ & 7.20 & 9.58 & 0.40 & 0.04 & 90.33 & 1515.01 \\
\hline $\mathrm{T}_{3} \mathrm{G}_{3}$ & 8.29 & 13.84 & 0.22 & 0.07 & 88.33 & 1955.78 \\
\hline $\mathrm{T}_{3} \mathrm{G}_{4}$ & 8.59 & 14.71 & 0.15 & 0.05 & 89.00 & 2075.33 \\
\hline $\mathrm{T}_{3} \mathrm{G}_{5}$ & 8.28 & 15.65 & 0.14 & 0.06 & 89.67 & 2144.90 \\
\hline $\mathrm{T}_{3} \mathrm{G}_{6}$ & 7.16 & 10.09 & 0.16 & 0.08 & 91.33 & 1575.38 \\
\hline $\mathrm{T}_{3} \mathrm{G}_{7}$ & 9.06 & 9.51 & 0.11 & 0.04 & 91.33 & 1695.01 \\
\hline $\mathrm{T}_{3} \mathrm{G}_{8}$ & 7.27 & 11.44 & 0.15 & 0.06 & 90.00 & 1685.12 \\
\hline $\mathrm{T}_{3} \mathrm{G}_{9}$ & 11.68 & 19.11 & 0.17 & 0.04 & 91.67 & 2821.88 \\
\hline $\mathrm{T}_{3} \mathrm{G}_{10}$ & 12.97 & 19.94 & 0.34 & 0.01 & 91.33 & 3003.39 \\
\hline $\mathrm{T}_{4} \mathrm{G}_{1}$ & 8.93 & 20.83 & 0.15 & 0.06 & 90.67 & 2697.18 \\
\hline $\mathrm{T}_{4} \mathrm{G}_{2}$ & 9.17 & 11.75 & 0.38 & 0.05 & 92.00 & 1926.89 \\
\hline $\mathrm{T}_{4} \mathrm{G}_{3}$ & 8.12 & 13.52 & 0.20 & 0.05 & 91.00 & 1964.60 \\
\hline $\mathrm{T}_{4} \mathrm{G}_{4}$ & 8.35 & 14.48 & 0.17 & 0.05 & 91.33 & 2084.28 \\
\hline $\mathrm{T}_{4} \mathrm{G}_{5}$ & 8.24 & 16.25 & 0.14 & 0.05 & 91.67 & 2246.01 \\
\hline $\mathrm{T}_{4} \mathrm{G}_{6}$ & 7.03 & 9.26 & 0.19 & 0.05 & 91.67 & 1494.59 \\
\hline
\end{tabular}




\begin{tabular}{|l|c|c|c|c|c|c|}
\hline $\mathrm{T}_{4} \mathrm{G}_{7}$ & 8.49 & 9.51 & 0.10 & 0.13 & 90.67 & 1634.22 \\
\hline $\mathrm{T}_{4} \mathrm{G}_{8}$ & 7.77 & 11.52 & 0.15 & 0.04 & 90.67 & 1759.74 \\
\hline $\mathrm{T}_{4} \mathrm{G}_{9}$ & 11.54 & 18.75 & 0.15 & 0.05 & 91.33 & 2764.78 \\
\hline $\mathrm{T}_{4} \mathrm{G}_{10}$ & 15.68 & 14.10 & 0.40 & 0.08 & 92.67 & 3064.73 \\
\hline $\mathrm{T}_{5} \mathrm{G}_{1}$ & 8.59 & 20.68 & 0.13 & 0.07 & 88.00 & 2577.61 \\
\hline $\mathrm{T}_{5} \mathrm{G}_{2}$ & 8.84 & 12.88 & 0.50 & 0.05 & 90.00 & 1954.80 \\
\hline $\mathrm{T}_{5} \mathrm{G}_{3}$ & 8.01 & 13.34 & 0.19 & 0.07 & 91.00 & 1943.07 \\
\hline $\mathrm{T}_{5} \mathrm{G}_{4}$ & 8.00 & 14.11 & 0.16 & 0.05 & 91.67 & 2025.62 \\
\hline $\mathrm{T}_{5} \mathrm{G}_{5}$ & 8.36 & 15.91 & 0.15 & 0.06 & 89.00 & 2159.39 \\
\hline $\mathrm{T}_{5} \mathrm{G}_{6}$ & 7.47 & 10.13 & 0.19 & 0.05 & 91.67 & 1612.81 \\
\hline $\mathrm{T}_{5} \mathrm{G}_{7}$ & 8.29 & 9.35 & 0.09 & 0.05 & 90.67 & 1598.37 \\
\hline $\mathrm{T}_{5} \mathrm{G}_{8}$ & 7.91 & 11.39 & 0.14 & 0.04 & 89.00 & 1718.86 \\
\hline $\mathrm{T}_{5} \mathrm{G}_{9}$ & 11.17 & 18.52 & 0.15 & 0.04 & 92.00 & 2732.08 \\
\hline $\mathrm{T}_{5} \mathrm{G}_{10}$ & 12.47 & 19.96 & 0.33 & 0.08 & 90.67 & 2944.03 \\
\hline SEm(土) & $\mathbf{0 . 1 7 7}$ & $\mathbf{0 . 1 3 2}$ & $\mathbf{0 . 0 1 2}$ & $\mathbf{0 . 0 0 9}$ & $\mathbf{0 . 0 6 3}$ & $\mathbf{1 8 . 5 7}$ \\
\hline LSD(0.05) & $\mathbf{0 . 5 0 1}$ & $\mathbf{0 . 4 0 3}$ & $\mathbf{0 . 0 3 7}$ & $\mathbf{0 . 0 2 8}$ & $\mathbf{0 . 1 9 1}$ & $\mathbf{5 5 . 2 8}$ \\
\hline
\end{tabular}

NB: Kailash- G1, Gobindabhog- G2, Kalma- G3, Dudheswar-G4, Malabati- G5, Kanakchur- G6, Kalojira- G7, Radhatilak- G8, IR36- $\mathrm{G}_{9}$ and Pusa-1509- $\mathrm{G}_{10}$. Earthen pot with seed moisture content $10 \%-\mathrm{T}_{1}$, cloth bag with seed moisture content $10 \%-\mathrm{T}_{2}$, aluminum foil packet with seed moisture content $10 \%-\mathrm{T}_{3}$, aluminum foil packet with seed moisture content $8 \%-\mathrm{T}_{4}$, and polythene packet with seed moisture content $10 \%-\mathrm{T}_{5}$.

At three months after storage, the longest seedling shoot length was found for $\mathrm{T}_{1} \mathrm{G}_{10}$ $(15.68 \mathrm{~cm})$ and it was shortest for $\mathrm{T}_{2} \mathrm{G}_{6}(5.64 \mathrm{~cm})$. In the case of root length $\mathrm{T}_{3} \mathrm{G}_{1}(20.95 \mathrm{~cm})$ recorded as highest and $\mathrm{T}_{2} \mathrm{G}_{2}(7.79 \mathrm{~cm})$ as lowest performed for this character. The fresh weight of seedling was the highest for $\mathrm{T}_{1} \mathrm{G}_{2}(0.41 \mathrm{~g})$ and lowest for $\mathrm{T}_{5} \mathrm{G}_{1}, \mathrm{~T}_{2} \mathrm{G}_{7} \& \mathrm{~T}_{1} \mathrm{G}_{8}(0.13 \mathrm{~g})$. Seedling dry weight was recorded as highest for $\mathrm{T}_{4} \mathrm{G}_{7}(0.13 \mathrm{~g})$ and lowest for $\mathrm{T}_{3} \mathrm{G}_{10}(0.01 \mathrm{~g})$. Maximum germination percentage (93.00) was recorded for $\mathrm{T}_{1} \mathrm{G}_{5}$ and it was lowest (88.00) for $\mathrm{T}_{5} \mathrm{G}_{1}$. But for the vigor index, it was high as (3064.73) for $\mathrm{T}_{4} \mathrm{G}_{10}$ and (1257.41) as lowest for $\mathrm{T}_{2} \mathrm{G}_{2}$.

So, it can be concluded that $\mathrm{T}_{1} \mathrm{G}_{5}$ (Malabati stored in earthen pot with seed moisture content $10 \%$ ) exhibited the highest germination percentage followed by $\mathrm{T}_{1} \mathrm{G}_{10}, \mathrm{~T}_{5} \mathrm{G}_{9}$, and $\mathrm{T}_{4} \mathrm{G}_{2}$ at three months after storage and $\mathrm{T}_{4} \mathrm{G}_{10}$ (Pusa-1509 stored in an aluminum foil packet with seed moisture content $8 \%$ ) recorded highest vigor index followed by $T_{3} G_{10}$ and $T_{2} G_{9}$ in comparison to other combination.

Table 3. Mean values with standard deviation for seedling shoot length, root length, fresh weight, dry weight, germination percentage, and vigor index of storage containers at three months after storage

\begin{tabular}{|l|l|c|c|c|c|c|c|}
\hline Treatments & $\begin{array}{c}\text { No of } \\
\text { Geno }\end{array}$ & $\begin{array}{c}\text { Shoot } \\
\text { length }(c m) \\
\text { Mean } \pm \text { SD }\end{array}$ & $\begin{array}{c}\text { Root length } \\
(c m) \\
\text { Mean } \pm \text { SD }\end{array}$ & $\begin{array}{c}\text { Fresh } \\
\text { weight }(g) \\
\text { Mean } \pm \text { SD }\end{array}$ & $\begin{array}{c}\text { Dry weight } \\
(\mathrm{g}) \text { Mean } \pm \text { SD }\end{array}$ & $\begin{array}{c}\text { Germination } \\
(\%) \\
\text { Mean } \pm \text { SD }\end{array}$ & GI Mean \pm SD \\
\hline $\mathrm{T}_{1}$ & 10 & $9.57^{\mathrm{A}} \pm 3.02$ & $13.41^{\mathrm{A}} \pm 4.06$ & $0.60^{\mathrm{A}} \pm 1.11$ & $0.06^{\mathrm{A}} \pm 0.016$ & $90.9^{\mathrm{A}} \pm 1.40$ & $2126.14^{\mathrm{A}} \pm 633.91$ \\
\hline $\mathrm{T}_{2}$ & 10 & $9.09^{\mathrm{A}} \pm 2.91$ & $13.90^{\mathrm{A}} \pm 4.36$ & $0.25^{\mathrm{A}} \pm 0.20$ & $0.054^{\mathrm{A}} \pm 0.022$ & $90.46^{\mathrm{A}} \pm 0.98$ & $2080.31^{\mathrm{A}} \pm 642.66$ \\
\hline $\mathrm{T}_{3}$ & 10 & $8.90^{\mathrm{A}} \pm 1.94$ & $14.48^{\mathrm{A}} \pm 4.37$ & $0.28^{\mathrm{A}} \pm 0.24$ & $0.051^{\mathrm{A}} \pm 0.019$ & $90.33^{\mathrm{A}} \pm 1.11$ & $2113.33^{\mathrm{A}} \pm 540.34$ \\
\hline $\mathrm{T}_{4}$ & 10 & $9.05^{\mathrm{A}} \pm 1.78$ & $14.58^{\mathrm{A}} \pm 4.23$ & $0.27^{\mathrm{A}} \pm 0.24$ & $0.094^{\mathrm{A}} \pm 0.119$ & $91.23^{\mathrm{A}} \pm 0.47$ & $2156.74^{\mathrm{A}} \pm 509.55$ \\
\hline
\end{tabular}




\begin{tabular}{|l|l|c|c|c|c|c|c|}
\hline $\mathrm{T}_{5}$ & 10 & $8.91^{\mathrm{A}} \pm 1.60$ & $14.62^{\mathrm{A}} \pm 4.02$ & $0.28^{\mathrm{A}} \pm 0.23$ & $0.056^{\mathrm{A}} \pm 0.013$ & $90.36^{\mathrm{A}} \pm 1.34$ & $2126.66^{\mathrm{A}} \pm 473.98$ \\
\hline SEm ( \pm ) & $\mathbf{0 . 3 4 7}$ & $\mathbf{0 . 6 2 7}$ & $\mathbf{0 . 0 7 8}$ & $\mathbf{0 . 1 6 4}$ & $\mathbf{0 . 1 6 5}$ & $\mathbf{1 3 . 0 1}$ \\
\hline LSD (0.05) & NS & NS & NS & NS & NS & NS \\
\hline
\end{tabular}

NB: Earthen pot with seed moisture content $10 \%-\mathrm{T}_{1}$, cloth bag with seed moisture content $10 \%-\mathrm{T}_{2}$, aluminum foil packet- $\mathrm{T}_{3}$, aluminum foil packet with seed moisture content $8 \%-\mathrm{T}_{4}$, and polythene packet with seed moisture content $10 \%-\mathrm{T}_{5}$.

At three months after storage, variation in the potentiality of storage containers for seedling shoot length, root length, vigor index, dry weight, and germination percentage were non-significant. All the genotypes belonged under a single group for all the characters studied. The mean value of shoot length was ranged from $8.90 \mathrm{~cm}$ to $9.57 \mathrm{~cm}$, and $\mathrm{T}_{1}$ and $\mathrm{T}_{3}$ recorded as the highest and lowest values respectively. The deviation from the mean value ranged from 1.60 to 3.02. The average longest root $(14.62 \mathrm{~cm})$ was found for $T_{5}$ and it was shortest for $T_{1}$ $(13.41 \mathrm{~cm})$. The deviation from the mean value was 4.02 to 4.37 . In the case of seedling fresh weight, maximum value $(0.60 \mathrm{~g})$ was recorded for $\mathrm{T}_{1}$ and lowest $(0.25 \mathrm{~g})$ for $\mathrm{T}_{2}$. The deviation from the mean value ranged from 0.20 to 1.11 and $\mathrm{T}_{1}$ showed the highest. Maximum seedling dry weight $(0.094 \mathrm{~g})$ was noted for $\mathrm{T}_{4}$ and it was lowest $(0.051 \mathrm{~g})$ for $\mathrm{T}_{3}$. The deviation from the mean value ranged from 0.016 to 0.119 . The mean value of germination percentage was as maximum as (91.23) for $\mathrm{T}_{4}$ and as minimum as $(90.33)$ for $\mathrm{T}_{3}$. With the deviation ranging from 0.47 to 1.40 and the highest vigor index (2156.74) was determined after $\mathrm{T}_{4}$ and it was the lowest (2080.31) for $\mathrm{T}_{2}$. The deviation from the mean value ranged from 473.98 to 642.66.

At three months after storage, $\mathrm{T}_{4}$ (aluminum foil packet with seed moisture content $8 \%$ ), Average over genotypes, recorded both highest germination $(\%)$ and vigor index followed by $\mathrm{T}_{1}$ and $\mathrm{T}_{2}$ for germination (\%) and $\mathrm{T}_{5}$ and $\mathrm{T}_{4}$ for vigor index. So, at three months after storage $\mathrm{T}_{4}$ (aluminum foil packet with seed moisture content $8 \%$ ) is best.

Table 4. Mean values for seedling shoot length, root length, fresh weight, dry weight, germination (\%), and vigor index of the genotypes stored in different containers at six months after storage

\begin{tabular}{|l|c|c|c|c|c|c|}
\hline $\begin{array}{c}\text { Genotypes with } \\
\text { treatments }\end{array}$ & $\begin{array}{c}\text { Shoot } \\
\text { length } \\
(\mathrm{cm})\end{array}$ & $\begin{array}{c}\text { Root } \\
\text { length } \\
(\mathrm{cm})\end{array}$ & $\begin{array}{c}\text { Fresh } \\
\text { weight }(\mathrm{g})\end{array}$ & $\begin{array}{c}\text { Dry } \\
\text { weight }(\mathrm{g})\end{array}$ & $\begin{array}{c}\text { Germination } \\
(\%)\end{array}$ & $\begin{array}{c}\text { Vigor } \\
\text { index }\end{array}$ \\
\hline $\mathrm{T}_{1} \mathrm{G}_{1}$ & 9.55 & 17.68 & 0.13 & 0.06 & 88.33 & 2400.67 \\
\hline $\mathrm{T}_{1} \mathrm{G}_{2}$ & 5.42 & 8.15 & 0.11 & 0.03 & 90.67 & 1250.19 \\
\hline $\mathrm{T}_{1} \mathrm{G}_{3}$ & 12.22 & 15.11 & 0.17 & 0.07 & 90.00 & 2460.54 \\
\hline $\mathrm{T}_{1} \mathrm{G}_{4}$ & 8.57 & 10.74 & 0.17 & 0.03 & 91.33 & 1767.24 \\
\hline $\mathrm{T}_{1} \mathrm{G}_{5}$ & 8.26 & 14.22 & 0.18 & 0.04 & 88.00 & 1979.02 \\
\hline $\mathrm{T}_{1} \mathrm{G}_{6}$ & 6.59 & 7.98 & 0.20 & 0.02 & 89.00 & 1297.86 \\
\hline $\mathrm{T}_{1} \mathrm{G}_{7}$ & 7.08 & 10.20 & 0.14 & 0.02 & 89.33 & 1538.08 \\
\hline $\mathrm{T}_{1} \mathrm{G}_{8}$ & 6.67 & 9.46 & 0.12 & 0.03 & 89.67 & 1442.72 \\
\hline $\mathrm{T}_{1} \mathrm{G}_{9}$ & 8.88 & 16.96 & 0.17 & 0.04 & 91.00 & 2350.69 \\
\hline $\mathrm{T}_{1} \mathrm{G}_{10}$ & 14.18 & 15.18 & 0.36 & 0.05 & 91.00 & 2673.90 \\
\hline $\mathrm{T}_{2} \mathrm{G}_{1}$ & 8.14 & 14.59 & 0.31 & 0.06 & 89.00 & 2022.72 \\
\hline $\mathrm{T}_{2} \mathrm{G}_{2}$ & 5.03 & 7.70 & 0.27 & 0.11 & 89.33 & 1140.47 \\
\hline $\mathrm{T}_{2} \mathrm{G}_{3}$ & 12.12 & 16.85 & 0.19 & 0.07 & 88.67 & 2559.49 \\
\hline $\mathrm{T}_{2} \mathrm{G}_{4}$ & 6.57 & 11.88 & 0.28 & 0.05 & 90.33 & 1664.89 \\
\hline
\end{tabular}




\begin{tabular}{|c|c|c|c|c|c|c|}
\hline $\mathrm{T}_{2} \mathrm{G}_{5}$ & 8.98 & 16.84 & 0.23 & 0.05 & 90.00 & 2316.53 \\
\hline $\mathrm{T}_{2} \mathrm{G}_{6}$ & 5.07 & 7.87 & 0.28 & 0.09 & 89.33 & 1158.40 \\
\hline $\mathrm{T}_{2} \mathrm{G}_{7}$ & 5.02 & 6.60 & 0.10 & 0.02 & 88.33 & 1025.59 \\
\hline $\mathrm{T}_{2} \mathrm{G}_{8}$ & 5.91 & 10.24 & 0.15 & 0.04 & 90.00 & 1453.53 \\
\hline $\mathrm{T}_{2} \mathrm{G}_{9}$ & 12.98 & 16.47 & 0.13 & 0.04 & 90.67 & 2670.40 \\
\hline $\mathrm{T}_{2} \mathrm{G}_{10}$ & 12.45 & 16.95 & 0.18 & 0.07 & 91.33 & 2684.50 \\
\hline $\mathrm{T}_{3} \mathrm{G}_{1}$ & 7.48 & 17.85 & 0.11 & 0.04 & 89.67 & 2268.26 \\
\hline $\mathrm{T}_{3} \mathrm{G}_{2}$ & 6.75 & 10.78 & 0.38 & 0.04 & 88.33 & 1548.43 \\
\hline $\mathrm{T}_{3} \mathrm{G}_{3}$ & 7.12 & 12.38 & 0.15 & 0.07 & 89.33 & 1744.18 \\
\hline $\mathrm{T}_{3} \mathrm{G}_{4}$ & 7.88 & 13.85 & 0.15 & 0.07 & 88.33 & 1920.46 \\
\hline $\mathrm{T}_{3} \mathrm{G}_{5}$ & 8.01 & 15.29 & 0.08 & 0.03 & 88.67 & 2068.04 \\
\hline $\mathrm{T}_{3} \mathrm{G}_{6}$ & 6.68 & 9.48 & 0.26 & 0.02 & 88.00 & 1422.46 \\
\hline $\mathrm{T}_{3} \mathrm{G}_{7}$ & 8.01 & 8.85 & 0.10 & 0.04 & 88.00 & 1483.66 \\
\hline $\mathrm{T}_{3} \mathrm{G}_{8}$ & 7.50 & 10.58 & 0.31 & 0.10 & 90.00 & 1626.36 \\
\hline $\mathrm{T}_{3} \mathrm{G}_{9}$ & 11.28 & 18.74 & 0.18 & 0.03 & 91.67 & 2751.47 \\
\hline $\mathrm{T}_{3} \mathrm{G}_{10}$ & 12.21 & 18.54 & 0.40 & 0.12 & 91.67 & 2823.17 \\
\hline $\mathrm{T}_{4} \mathrm{G}_{1}$ & 8.29 & 10.42 & 0.28 & 0.05 & 88.00 & 1637.53 \\
\hline $\mathrm{T}_{4} \mathrm{G}_{2}$ & 6.03 & 9.26 & 0.14 & 0.04 & 88.33 & 1351.46 \\
\hline $\mathrm{T}_{4} \mathrm{G}_{3}$ & 7.79 & 12.52 & 0.16 & 0.03 & 88.33 & 1790.72 \\
\hline $\mathrm{T}_{4} \mathrm{G}_{4}$ & 7.57 & 15.25 & 0.14 & 0.05 & 89.33 & 2039.80 \\
\hline $\mathrm{T}_{4} \mathrm{G}_{5}$ & 7.49 & 9.37 & 0.11 & 0.03 & 89.33 & 1506.12 \\
\hline $\mathrm{T}_{4} \mathrm{G}_{6}$ & 8.35 & 14.48 & 0.17 & 0.05 & 89.33 & 2039.17 \\
\hline $\mathrm{T}_{4} \mathrm{G}_{7}$ & 12.32 & 19.42 & 0.29 & 0.08 & 86.67 & 2748.50 \\
\hline $\mathrm{T}_{4} \mathrm{G}_{8}$ & 7.89 & 11.52 & 0.15 & 0.04 & 87.67 & 1700.29 \\
\hline $\mathrm{T}_{4} \mathrm{G}_{9}$ & 8.93 & 20.18 & 0.15 & 0.06 & 90.33 & 2625.27 \\
\hline $\mathrm{T}_{4} \mathrm{G}_{10}$ & 11.54 & 18.75 & 0.15 & 0.05 & 91.00 & 2759.76 \\
\hline $\mathrm{T}_{5} \mathrm{G}_{1}$ & 7.92 & 20.68 & 0.11 & 0.07 & 88.00 & 2514.66 \\
\hline $\mathrm{T}_{5} \mathrm{G}_{2}$ & 8.14 & 12.88 & 0.29 & 0.05 & 87.67 & 1840.59 \\
\hline $\mathrm{T}_{5} \mathrm{G}_{3}$ & 7.84 & 13.34 & 0.19 & 0.07 & 87.67 & 1858.03 \\
\hline $\mathrm{T}_{5} \mathrm{G}_{4}$ & 7.07 & 14.11 & 0.16 & 0.05 & 89.00 & 1888.95 \\
\hline $\mathrm{T}_{5} \mathrm{G}_{5}$ & 7.36 & 15.91 & 0.15 & 0.06 & 88.33 & 2054.72 \\
\hline $\mathrm{T}_{5} \mathrm{G}_{6}$ & 7.80 & 10.15 & 0.19 & 0.05 & 88.33 & 1585.50 \\
\hline $\mathrm{T}_{5} \mathrm{G}_{7}$ & 8.29 & 9.35 & 0.29 & 0.05 & 88.00 & 1552.71 \\
\hline $\mathrm{T}_{5} \mathrm{G}_{8}$ & 7.91 & 11.39 & 0.14 & 0.04 & 87.67 & 1692.24 \\
\hline $\mathrm{T}_{5} \mathrm{G}_{9}$ & 11.18 & 18.52 & 0.15 & 0.04 & 90.00 & 2671.24 \\
\hline $\mathrm{T}_{5} \mathrm{G}_{10}$ & 12.40 & 19.96 & 0.33 & 0.08 & 91.33 & 2951.48 \\
\hline $\operatorname{SEm}( \pm)$ & 0.172 & 0.128 & 0.012 & 0.009 & 0.051 & 17.76 \\
\hline $\operatorname{LSD}(0.05)$ & 0.501 & 0.399 & 0.036 & 0.026 & 0.152 & 51.77 \\
\hline
\end{tabular}

NB: Kailash- $\mathrm{G}_{1}$, Gobindabhog- G2, Kalma- G3, Dudheswar-G4, Malabati- G5, Kanakchur- G6, Kalojira- G7, Radhatilak- G8, IR36- $\mathrm{G}_{9}$ and Pusa-1509- $\mathrm{G}_{10}$. Earthen pot with seed moisture content $10 \%-\mathrm{T}_{1}$, cloth bag with seed moisture content $10 \%-\mathrm{T}_{2}$, aluminum foil packet with seed moisture content $10 \%-\mathrm{T}_{3}$, aluminum foil packet with seed moisture content $8 \%$ - $\mathrm{T}_{4}$, and polythene packet with seed moisture content $10 \%-\mathrm{T}_{5}$.

At six months after storage, the longest root was noted $(20.68 \mathrm{~cm})$ for $\mathrm{T}_{5} \mathrm{G}_{1}$ and it was lowest for $\mathrm{T}_{2} \mathrm{G}_{7}(6.60 \mathrm{~cm})$. Shoot length was highest for $\mathrm{T}_{1} \mathrm{G}_{10}(14.18 \mathrm{~cm})$ and lowest for $\mathrm{T}_{2} \mathrm{G}_{7}$ $(5.02 \mathrm{~cm})$. The highest fresh weight of seedling was observed for $\mathrm{T}_{3} \mathrm{G}_{10}(0.40 \mathrm{~g})$ and lowest for 
$\mathrm{T}_{3} \mathrm{G}_{5}(0.08 \mathrm{~g})$. While maximum dry weight $(0.12 \mathrm{~g})$ was noted for $\mathrm{T}_{3} \mathrm{G}_{10}$ and the lowest were found for $\mathrm{T}_{3} \mathrm{G}_{6} \mathrm{~T}_{2} \mathrm{G}_{7} \mathrm{~T}_{1} \mathrm{G}_{6}$ and $\mathrm{T}_{1} \mathrm{G}_{7}(0.02 \mathrm{~g})$. After six months of storage germination percentage was highest in both $\mathrm{T}_{3} \mathrm{G}_{10}$ and $\mathrm{T}_{3} \mathrm{G}_{9}$ (91.67) and lowest for $\mathrm{T}_{4} \mathrm{G}_{7}$ (86.67). Vigor index was recorded as maximum as (2951.48) for $\mathrm{T}_{5} \mathrm{G}_{10}$ and lowest for $\mathrm{T}_{2} \mathrm{G}_{2}$ (1140.47).

So, it can be concluded that $\mathrm{T}_{3} \mathrm{G}_{9}$ (IR-36 store in the aluminum foil packet) showed the highest germination percentage followed by $\mathrm{T}_{1} \mathrm{G}_{4}$ and $\mathrm{T}_{3} \mathrm{G}_{10}$ (Pusa-1509 stored in the aluminum foil packet) at six months after storage and $\mathrm{T}_{5} \mathrm{G}_{10}$ (Pusa-1509 stored in polythene packet with seed moisture content 10\%) recorded highest Vigor index followed by $\mathrm{T}_{4} \mathrm{G}_{7}$ and $\mathrm{T}_{3} \mathrm{G}_{10}$ in comparison to other combination.

Table 5. Mean Value with standard deviation for seedling shoot length, root length, fresh weight, dry weight, germination (\%) and vigor index of storage containers at six months after storage

\begin{tabular}{|c|c|c|c|c|c|c|c|}
\hline Treatments & $\begin{array}{c}\text { No } \\
\text { of } \\
\text { Geno }\end{array}$ & $\begin{array}{c}\text { Shoot } \\
\text { length } \\
(\mathrm{cm}) \\
\text { Mean } \pm \text { SD }\end{array}$ & $\begin{array}{l}\text { Root length } \\
\quad(\mathrm{cm}) \\
\text { Mean } \pm \text { SD }\end{array}$ & \begin{tabular}{|} 
Fresh weight \\
$(\mathrm{g})$ \\
Mean $\pm \mathrm{SD}$
\end{tabular} & $\begin{array}{l}\text { Dry weight }(\mathrm{g}) \\
\text { Mean } \pm \text { SD }\end{array}$ & $\begin{array}{c}\text { Germination } \% \\
\text { Mean } \pm \text { SD }\end{array}$ & GI Mean \pm SD \\
\hline $\mathrm{T}_{1}$ & 10 & $8.74^{\mathrm{A}} \pm 2.69$ & $12.56^{\mathrm{A}} \pm 3.66$ & $0.17^{\mathrm{B}} \pm 0.071$ & $0.039^{\mathrm{B}} \pm 0.016$ & $89.83^{\mathrm{A}} \pm 1.16$ & $1916.09^{\mathrm{A}} \pm 528.42$ \\
\hline $\mathrm{T}_{2}$ & 10 & $8.22^{\mathrm{A}} \pm 3.24$ & $12.59^{\mathrm{A}} \pm 4.24$ & $0.19^{\mathrm{AB}} \pm 0.072$ & $0.107^{\mathrm{A}} \pm 0.079$ & $89.69^{\mathrm{A}} \pm 0.93$ & $1869.65^{\mathrm{A}} \pm 664.10$ \\
\hline $\mathrm{T}_{3}$ & 10 & $8.29^{\mathrm{A}} \pm 1.89$ & $13.63^{\mathrm{A}} \pm 3.79$ & $0.33^{\mathrm{A}} \pm 0.281$ & $0.056^{\mathrm{AB}} \pm 0.033$ & $89.36^{\mathrm{AB}} \pm 1.39$ & $1965.64^{\mathrm{A}} \pm 507.99$ \\
\hline $\mathrm{T}_{4}$ & 10 & $8.59^{\mathrm{A}} \pm 1.74$ & $14.62^{\mathrm{A}} \pm 4.01$ & $0.23^{\mathrm{AB}} \pm 0.143$ & $0.056^{\mathrm{AB}} \pm 0.013$ & $88.60^{\mathrm{B}} \pm 1.20$ & $2061.01^{\mathrm{A}} \pm 484.31$ \\
\hline $\mathrm{T}_{5}$ & 10 & $8.62^{\mathrm{A}} \pm 1.90$ & $14.11^{\mathrm{A}} \pm 4.17$ & $0.25^{\mathrm{AB}} \pm 0.224$ & $0.088^{\mathrm{AB}} \pm 0.12$ & $88.83^{\mathrm{AB}} \pm 1.28$ & $2019.86^{\mathrm{A}} \pm 522.51$ \\
\hline \multicolumn{2}{|l|}{$\overline{\operatorname{SEm}( \pm)}$} & 0.353 & 0.594 & 0.026 & 0.010 & 0.179 & 81.265 \\
\hline \multicolumn{2}{|l|}{ LSD (0.05) } & NS & NS & NS & NS & NS & NS \\
\hline
\end{tabular}

NB: Earthen pot with seed moisture content $10 \%-\mathrm{T}_{1}$, cloth bag with seed moisture content $10 \%-\mathrm{T}_{2}$, aluminum foil packet- $\mathrm{T}_{3}$, aluminum foil packet with seed moisture content $8 \%-\mathrm{T}_{4}$, and polythene packet with seed moisture content $10 \%-\mathrm{T}_{5}$.

At six months after storage, treatments over genotypes shoot length was highest in $\mathrm{T}_{1}$ $(8.74 \mathrm{~cm})$ and $\mathrm{T}_{2},(8.22 \mathrm{~cm})$ was showed lowest. The deviation from the mean value was ranged from 1.74 to 3.24 and the $\mathrm{T}_{2}$ was the highest and all genotypes were non-significant in variation for this trait. Genotypes were grouped under the same group i.e A group. In the case of root length $\mathrm{T}_{5}(14.62 \mathrm{~cm})$ showed the highest and $\mathrm{T}_{1},(12.56 \mathrm{~cm})$ was showed the lowest and the deviation from the mean value was ranged from 3.66 to 4.24 and the $\mathrm{T}_{2}$ was showed the highest and all genotypes were non-significant variation. All genotypes were grouped under the same group i.e. under $\mathrm{A}$. In fresh weight $\mathrm{T}_{3},(0.33 \mathrm{~g})$ showed highest and $\mathrm{T}_{1}(0.17 \mathrm{~g})$ showed lowest. The deviation from the mean value ranged from 0.071 to 0.281 and $\mathrm{T}_{3}$ was highest and the genotypes showed non-significant variation $\mathrm{T}_{1}$, which was grouped under $\mathrm{B}, \mathrm{T}_{2}, \mathrm{~T}_{4}$, and $\mathrm{T}_{5}$ were grouped under $\mathrm{AB}, \mathrm{T}_{3}$ was grouped under $\mathrm{A}$. Dry weight was recorded highest in $\mathrm{T}_{2}(0.107 \mathrm{~g})$ and lowest in $\mathrm{T}_{1}$ was shows lowest $(0.039 \mathrm{~g})$. The deviation from the mean value ranged from 0.013 to 0.079 and $\mathrm{T}_{2}$ was showed highest and showed the non-significant variation. $\mathrm{T}_{1}$ was grouped under $\mathrm{B}, \mathrm{T}_{2}$ grouped under $\mathrm{A} . \mathrm{T}_{3}, \mathrm{~T}_{4}$, and $\mathrm{T}_{5}$ were grouped under $\mathrm{AB}$. Germination percentage was highest in $\mathrm{T}_{1}(89.83)$ and $\mathrm{T}_{5}$ lowest in (88.60). The deviation from the mean value ranged from 0.93 to 1.39 and $\mathrm{T}_{3}$ showed the highest. The treatments were non-significant variation. The treatments were grouped under two groups. $T_{1}$ and $T_{2}$ were grouped under $A . T_{3}$ 
and $\mathrm{T}_{4}$ were grouped under $\mathrm{A} \mathrm{B}, \mathrm{T}_{5}$ was grouped under $\mathrm{B}$ group. Among the genotypes, vigor index found the highest $\mathrm{T}_{4}$ (2061.01) and the lowest in $\mathrm{T}_{2}$ (1869.65). The deviation from the mean value ranged from 664.10 to $484.31 . T_{2}$ was shows highest. The treatments over genotypes were non-significant variation and all genotypes were grouped under A.

At six months after storage, $\mathrm{T}_{1}$ (earthen pot with seed moisture content $10 \%$ ) preferred best for germination percentage followed by $\mathrm{T}_{2} \& \mathrm{~T}_{3}$ while $\mathrm{T}_{4}$ (aluminum foil packet with seed moisture content $8 \%$ ), performed best for Vigor index followed by $\mathrm{T}_{5}$ and $\mathrm{T}_{3}$. So, at six months after storage $\mathrm{T}_{4}$ (aluminum foil packet with seed moisture content $8 \%$ ) could be identified as best with joint consideration of germination $(\%)$ and vigor index.

Table 6. Mean values for seedling shoot length, root length, fresh weight, dry weight, germination (\%) and vigor index of the genotypes stored in different containers at nine months after storage

\begin{tabular}{|c|c|c|c|c|c|c|}
\hline $\begin{array}{l}\text { Genotypes with } \\
\text { treatments }\end{array}$ & $\begin{array}{l}\text { Shoot } \\
\text { length } \\
(\mathrm{cm})\end{array}$ & $\begin{array}{l}\text { Root } \\
\text { length } \\
(\mathrm{cm})\end{array}$ & $\begin{array}{c}\text { Fresh } \\
\text { weight }(\mathrm{g})\end{array}$ & $\begin{array}{c}\text { Dry } \\
\text { weight }(\mathrm{g})\end{array}$ & $\begin{array}{c}\text { Germination } \\
(\%)\end{array}$ & $\begin{array}{l}\text { Vigor } \\
\text { index }\end{array}$ \\
\hline $\mathrm{T}_{1} \mathrm{G}_{1}$ & 9.22 & 17.68 & 0.13 & 0.06 & 88.33 & 2379.17 \\
\hline $\mathrm{T}_{1} \mathrm{G}_{2}$ & 5.29 & 8.15 & 0.11 & 0.03 & 88.00 & 1182.84 \\
\hline $\mathrm{T}_{1} \mathrm{G}_{3}$ & 12.22 & 15.11 & 0.13 & 0.07 & 87.67 & 2395.22 \\
\hline $\mathrm{T}_{1} \mathrm{G}_{4}$ & 7.93 & 10.76 & 0.14 & 0.03 & 88.67 & 1687.30 \\
\hline $\mathrm{T}_{1} \mathrm{G}_{5}$ & 7.93 & 14.03 & 0.06 & 0.04 & 87.67 & 1921.16 \\
\hline $\mathrm{T}_{1} \mathrm{G}_{6}$ & 7.25 & 7.98 & 0.20 & 0.02 & 88.00 & 1341.52 \\
\hline $\mathrm{T}_{1} \mathrm{G}_{7}$ & 6.91 & 9.93 & 0.14 & 0.02 & 87.67 & 1478.53 \\
\hline $\mathrm{T}_{1} \mathrm{G}_{8}$ & 6.67 & 9.29 & 0.11 & 0.03 & 86.33 & 1376.89 \\
\hline $\mathrm{T}_{1} \mathrm{G}_{9}$ & 8.88 & 17.96 & 0.17 & 0.04 & 90.00 & 2417.42 \\
\hline $\mathrm{T}_{1} \mathrm{G}_{10}$ & 13.87 & 15.07 & 0.36 & 0.05 & 89.33 & 2586.79 \\
\hline $\mathrm{T}_{2} \mathrm{G}_{1}$ & 8.04 & 14.51 & 0.31 & 0.06 & 89.00 & 2004.72 \\
\hline $\mathrm{T}_{2} \mathrm{G}_{2}$ & 5.03 & 7.70 & 0.27 & 0.04 & 87.67 & 1115.54 \\
\hline $\mathrm{T}_{2} \mathrm{G}_{3}$ & 9.14 & 16.74 & 0.20 & 0.02 & 87.33 & 2258.05 \\
\hline $\mathrm{T}_{2} \mathrm{G}_{4}$ & 6.50 & 11.81 & 0.28 & 0.08 & 88.00 & 1609.38 \\
\hline $\mathrm{T}_{2} \mathrm{G}_{5}$ & 8.91 & 16.80 & 0.22 & 0.03 & 87.33 & 2247.10 \\
\hline $\mathrm{T}_{2} \mathrm{G}_{6}$ & 5.07 & 7.87 & 0.11 & 0.03 & 88.33 & 1144.31 \\
\hline $\mathrm{T}_{2} \mathrm{G}_{7}$ & 4.71 & 6.53 & 0.10 & 0.02 & 87.33 & 981.48 \\
\hline $\mathrm{T}_{2} \mathrm{G}_{8}$ & 5.67 & 10.24 & 0.14 & 0.04 & 86.67 & 1378.59 \\
\hline $\mathrm{T}_{2} \mathrm{G}_{9}$ & 11.74 & 16.47 & 0.13 & 0.04 & 90.33 & 2518.17 \\
\hline $\mathrm{T}_{2} \mathrm{G}_{10}$ & 12.34 & 16.78 & 0.18 & 0.07 & 90.00 & 2622.17 \\
\hline $\mathrm{T}_{3} \mathrm{G}_{1}$ & 7.21 & 17.85 & 0.11 & 0.04 & 87.00 & 2178.13 \\
\hline $\mathrm{T}_{3} \mathrm{G}_{2}$ & 6.75 & 10.71 & 0.38 & 0.04 & 87.00 & 1519.48 \\
\hline $\mathrm{T}_{3} \mathrm{G}_{3}$ & 7.15 & 11.21 & 0.12 & 0.07 & 86.67 & 1592.63 \\
\hline $\mathrm{T}_{3} \mathrm{G}_{4}$ & 7.81 & 13.80 & 0.15 & 0.06 & 87.33 & 1886.77 \\
\hline $\mathrm{T}_{3} \mathrm{G}_{5}$ & 7.97 & 13.44 & 0.08 & 0.03 & 87.33 & 1868.30 \\
\hline
\end{tabular}




\begin{tabular}{|c|c|c|c|c|c|c|}
\hline $\mathrm{T}_{3} \mathrm{G}_{6}$ & 6.68 & 9.41 & 0.26 & 0.02 & 86.67 & 1395.95 \\
\hline $\mathrm{T}_{3} \mathrm{G}_{7}$ & 8.01 & 8.85 & 0.28 & 0.04 & 88.00 & 1482.49 \\
\hline $\mathrm{T}_{3} \mathrm{G}_{8}$ & 7.37 & 10.58 & 0.29 & 0.10 & 87.33 & 1567.36 \\
\hline $\mathrm{T}_{3} \mathrm{G}_{9}$ & 11.14 & 18.41 & 0.18 & 0.03 & 87.67 & 2592.99 \\
\hline $\mathrm{T}_{3} \mathrm{G}_{10}$ & 12.21 & 18.54 & 0.40 & 0.12 & 87.33 & 2685.01 \\
\hline $\mathrm{T}_{4} \mathrm{G}_{1}$ & 8.20 & 10.42 & 0.28 & 0.05 & 85.67 & 1593.48 \\
\hline $\mathrm{T}_{4} \mathrm{G}_{2}$ & 5.65 & 9.26 & 0.14 & 0.04 & 86.67 & 1289.22 \\
\hline $\mathrm{T}_{4} \mathrm{G}_{3}$ & 7.54 & 11.82 & 0.16 & 0.03 & 86.33 & 1667.16 \\
\hline $\mathrm{T}_{4} \mathrm{G}_{4}$ & 6.91 & 14.91 & 0.14 & 0.05 & 87.67 & 1913.34 \\
\hline $\mathrm{T}_{4} \mathrm{G}_{5}$ & 7.49 & 9.37 & 0.09 & 0.06 & 86.67 & 1462.78 \\
\hline $\mathrm{T}_{4} \mathrm{G}_{6}$ & 8.35 & 13.48 & 0.17 & 0.05 & 88.33 & 1928.32 \\
\hline $\mathrm{T}_{4} \mathrm{G}_{7}$ & 12.32 & 17.42 & 0.29 & 0.08 & 87.00 & 2590.28 \\
\hline $\mathrm{T}_{4} \mathrm{G}_{8}$ & 7.89 & 11.52 & 0.15 & 0.04 & 87.00 & 1687.76 \\
\hline $\mathrm{T}_{4} \mathrm{G}_{9}$ & 8.93 & 18.51 & 0.15 & 0.06 & 88.00 & 2415.18 \\
\hline $\mathrm{T}_{4} \mathrm{G}_{10}$ & 11.54 & 16.09 & 0.15 & 0.05 & 88.00 & 2434.51 \\
\hline $\mathrm{T}_{5} \mathrm{G}_{1}$ & 7.59 & 16.34 & 0.11 & 0.07 & 86.33 & 2068.93 \\
\hline $\mathrm{T}_{5} \mathrm{G}_{2}$ & 8.14 & 12.21 & 0.29 & 0.04 & 86.33 & 1754.77 \\
\hline $\mathrm{T}_{5} \mathrm{G}_{3}$ & 7.84 & 12.74 & 0.19 & 0.06 & 87.67 & 1804.47 \\
\hline $\mathrm{T}_{5} \mathrm{G}_{4}$ & 7.03 & 13.11 & 0.15 & 0.05 & 85.67 & 1725.30 \\
\hline $\mathrm{T}_{5} \mathrm{G}_{5}$ & 7.36 & 13.91 & 0.15 & 0.06 & 86.00 & 1828.77 \\
\hline $\mathrm{T}_{5} \mathrm{G}_{6}$ & 7.50 & 10.15 & 0.19 & 0.04 & 85.00 & 1502.26 \\
\hline $\mathrm{T}_{5} \mathrm{G}_{7}$ & 8.29 & 9.35 & 0.27 & 0.05 & 87.33 & 1542.29 \\
\hline $\mathrm{T}_{5} \mathrm{G}_{8}$ & 7.91 & 11.39 & 0.14 & 0.04 & 88.33 & 1704.23 \\
\hline $\mathrm{T}_{5} \mathrm{G}_{9}$ & 10.18 & 15.86 & 0.15 & 0.04 & 88.33 & 2301.62 \\
\hline $\mathrm{T}_{5} \mathrm{G}_{10}$ & 12.40 & 16.74 & 0.33 & 0.08 & 89.33 & 2603.05 \\
\hline $\operatorname{SEm}( \pm)$ & 0.169 & 0.123 & 0.011 & 0.008 & 0.054 & 17.64 \\
\hline $\operatorname{LSD}(0.05)$ & 0.495 & 0.379 & 0.035 & 0.023 & 0.150 & 52.85 \\
\hline
\end{tabular}

NB: Kailash- $\mathrm{G}_{1}$, Gobindabhog- $\mathrm{G}_{2}$, Kalma- $\mathrm{G}_{3}$, Dudheswar-G4, Malabati- G5, Kanakchur- G6, Kalojira- G7, Radhatilak- G8, IR36- $\mathrm{G}_{9}$ and Pusa-1509- $\mathrm{G}_{10}$. Earthen pot with seed moisture content $10 \%-\mathrm{T}_{1}$, cloth bag with seed moisture content $10 \%-\mathrm{T}_{2}$, aluminum foil packet with seed moisture content $10 \%-\mathrm{T}_{3}$, aluminum foil packet with seed moisture content $8 \%$ - $\mathrm{T}_{4}$, and polythene packet with seed moisture content $10 \%-\mathrm{T}_{5}$.

After nine months of seed storage of ten genotypes in a different containers, maximum shoot length was observed for $\mathrm{T}_{1} \mathrm{G}_{10}(13.87 \mathrm{~cm})$ and minimum for $\mathrm{T}_{2} \mathrm{G}_{7}(4.71 \mathrm{~cm})$. Root length was maximum for $\mathrm{T}_{3} \mathrm{G}_{10}(18.54 \mathrm{~cm})$ and minimum for $\mathrm{T}_{2} \mathrm{G}_{7}(6.53 \mathrm{~cm})$. Fresh weight was seen as highest for $\mathrm{T}_{3} \mathrm{G}_{10}(0.40 \mathrm{~g})$ and lowest for $\mathrm{T}_{1} \mathrm{G}_{5}(0.06 \mathrm{~g})$. The average seedling dry weight was recorded as maximum for $\mathrm{T}_{3} \mathrm{G}_{10}(0.12 \mathrm{~g})$ and lowest for $\mathrm{T}_{1} \mathrm{G}_{6}, \mathrm{~T}_{1} \mathrm{G}_{7}, \mathrm{~T}_{2} \mathrm{G}_{7}(0.02 \mathrm{~g})$. Average germination percentage was highest for $\mathrm{T}_{2} \mathrm{G}_{9}$ (90.33) and lowest for $\mathrm{T}_{5} \mathrm{G}_{6}$ (85.00). Vigor index 
was recorded as maximum for $\mathrm{T}_{3} \mathrm{G}_{10}$ (2685.01) followed by $\mathrm{T}_{2} \mathrm{G}_{10}$ (2622.17), $\mathrm{T}_{5} \mathrm{G}_{10}(2603.00)$, $\mathrm{T}_{3} \mathrm{G}_{9}$ (2592.99), and $\mathrm{T}_{4} \mathrm{G}_{7}$ (2590.28).

So, it can be concluded that $\mathrm{T}_{2} \mathrm{G}_{9}$ (IR-36 stored in a cloth bag with seed moisture content $10 \%$ ) was recorded highest germination percentage followed by $\mathrm{T}_{1} \mathrm{G}_{9}$ and $\mathrm{T}_{2} \mathrm{G}_{10}$ at nine months after storage and $\mathrm{T}_{3} \mathrm{G}_{10}$ (Pusa-1509 stored with aluminum foil packet), recorded highest vigor index followed by $\mathrm{T}_{2} \mathrm{G}_{10}$ and $\mathrm{T}_{5} \mathrm{G}_{10}$.

Table 7. Mean value with a standard deviation of shoot length, root length, fresh weight, dry weight, germination (\%) and vigor index of seed stored in different containers after nine months after storage

\begin{tabular}{|c|c|c|c|c|c|c|c|}
\hline Treatments & $\begin{array}{c}\text { No of } \\
\text { Genotypes }\end{array}$ & $\begin{array}{c}\text { Shoot } \\
\text { length } \\
(\mathrm{cm}) \\
\text { Mean } \pm \text { SD }\end{array}$ & $\begin{array}{l}\text { Root length } \\
(\mathrm{cm}) \\
\text { Mean } \pm \text { SD }\end{array}$ & $\begin{array}{l}\text { Fresh weight } \\
\text { (g) Mean } \pm \text { SD }\end{array}$ & $\begin{array}{l}\text { Dry weight } \\
\text { (g) Mean } \pm \text { SD }\end{array}$ & $\begin{array}{c}\text { Germination } \% \\
\text { Mean } \pm \text { SD }\end{array}$ & GI Mean \pm SD \\
\hline $\mathrm{T}_{1}$ & 10 & $8.61^{\mathrm{A}} \pm 2.61$ & $12.59^{\mathrm{A}} \pm 3.82$ & $0.155^{\mathrm{B}} \pm 0.081$ & $0.039^{\mathrm{B}} \pm 0.016$ & $88.16^{\mathrm{A}} \pm 1.009$ & $1876.68^{\mathrm{A}} \pm 530.40$ \\
\hline $\mathrm{T}_{2}$ & 10 & $7.71^{\mathrm{A}} \pm 2.78$ & $12.54^{\mathrm{A}} \pm 4.21$ & $0.194^{\mathrm{AB}} \pm 0.074$ & $0.106^{\mathrm{A}} \pm 0.079$ & $88.19^{\mathrm{A}} \pm 1.218$ & $1787.95^{\mathrm{A}} \pm 616.82$ \\
\hline $\mathrm{T}_{3}$ & 10 & $8.23^{\mathrm{A}} \pm 1.88$ & $13.28^{\mathrm{A}} \pm 3.77$ & $0.334^{\mathrm{A}} \pm 0.283$ & $0.055^{\mathrm{AB}} \pm 0.032$ & $87.23^{\mathrm{B}} \pm 0.416$ & $1876.91^{\mathrm{A}} \pm 465.23$ \\
\hline $\mathrm{T}_{4}$ & 10 & $8.48^{\mathrm{A}} \pm 2.03$ & $13.28^{\mathrm{A}} \pm 3.33$ & $0.25^{\mathrm{AB}} \pm 0.224$ & $0.081^{\mathrm{AB}} \pm 0.098$ & $87.13^{\mathrm{B}} \pm 0.848$ & $1898.20^{\mathrm{A}} \pm 445.46$ \\
\hline $\mathrm{T}_{5}$ & 10 & $8.42^{\mathrm{A}} \pm 1.64$ & $13.18^{\mathrm{A}} \pm 2.55$ & $0.229^{\mathrm{AB}} \pm 0.144$ & $0.053^{\mathrm{AB}} \pm 0.014$ & $87.03^{\mathrm{B}} \pm 1.381$ & $1883.56^{\mathrm{A}} \pm 345.07$ \\
\hline \multicolumn{2}{|l|}{$\operatorname{SEm}( \pm)$} & 0.333 & 0.534 & 0.026 & 8.819 & 0.153 & 72.90 \\
\hline \multicolumn{2}{|l|}{ LSD (0.05) } & NS & NS & NS & NS & 0.9278* & NS \\
\hline
\end{tabular}

NB: Earthen pot with seed moisture content $10 \%-\mathrm{T}_{1}$, cloth bag with seed moisture content $10 \%-\mathrm{T}_{2}$, aluminum foil packet- $\mathrm{T}_{3}$, aluminum foil packet with seed moisture content $8 \%-\mathrm{T}_{4}$, and polythene packet with seed moisture content $10 \%-\mathrm{T}_{5}$.

At nine months after storage treatments over genotypes shoot length was highest in $\mathrm{T}_{1}$ (8.61) and lowest in $T_{2}(7.71 \mathrm{~cm})$. The deviation from the mean value ranged from 1.64 to 2.78 and $\mathrm{T}_{2}$ was the highest. All treatments over genotypes showed non-significant variation for this trait and were grouped under the same $A$. In the case of root length $T_{3} \& T_{4}$ (13.28) showed the highest and $\mathrm{T}_{2}$ (12.54) showed the lowest. The deviation from the mean value ranged from 2.55 to 4.21 and $\mathrm{T}_{2}$ was the highest. All genotypes were non-significant variation and were grouped under the same A. Highest fresh weight was found in $\mathrm{T}_{3}(0.334 \mathrm{~g})$ and lowest in $\mathrm{T}_{1}(0.155 \mathrm{~g})$. The deviation from the mean value was ranged from 0.074 to 0.283 and $\mathrm{T}_{3}$ showed the highest. The genotypes were non-significant variation. $\mathrm{T}_{1}$ was grouped under $\mathrm{B}, \mathrm{T}_{2}, \mathrm{~T}_{4}$, and $\mathrm{T}_{5}$ were grouped under $A B, T_{3}$ was grouped under $A$. Dry weight was the highest $T_{2}(0.106 g)$ and the lowest in $T_{1}$ $(0.039 \mathrm{~g})$. The deviation from the mean value was ranged from 0.014 to 0.098 and $\mathrm{T}_{4}$ was the highest genotypes were non-significant variation. $\mathrm{T}_{1}$ was grouped under $\mathrm{B}, \mathrm{T}_{2}$ was grouped under $A, T_{3}, T_{4}$, and $T_{5}$ were grouped under $A B$. Germination percentage was found in $T_{2}$ (88.19) and lowest in $T_{5}$ (87.03). The deviation from the mean value was ranged from 0.416 to 1.381 and $\mathrm{T}_{5}$ was the highest. The genotypes were significant variation. $\mathrm{T}_{1}$ and $\mathrm{T}_{2}$ were grouped under $\mathrm{A}, \mathrm{T}_{3}, \mathrm{~T}_{4}$, and $\mathrm{T}_{5}$ were grouped under $\mathrm{B}$. Among the genotypes, highest vigor index was recorded in $\mathrm{T}_{4}(1898.20)$ and the lowest in $\mathrm{T}_{2}$ (1787.95). The deviation from the mean value was ranged from 345.07 to 616.82 and $\mathrm{T}_{2}$ was the highest. The genotypes showed non-significant variation they were grouped under the same group i.e. under A.

At nine months after storage, the seed of genotypes stored in $\mathrm{T}_{2}$ (cloth bag with seed moisture content $10 \%$ ) maintained the highest germination percentage followed by $\mathrm{T}_{3}$ and $\mathrm{T}_{1}$ and 
while highest vigor status of seed was maintained when stored in $\mathrm{T}_{4}$ (aluminum foil packet with seed moisture content $8 \%$ ) followed by $\mathrm{T}_{5}$ and $\mathrm{T}_{3}$. Aluminum foil packet with seed moisture content $8 \%$ therefore, can be recorded as best storage materials when the seed of this crop, in general, are stored for nine months.

Table 8. Mean values for seedling shoot length, root length, fresh weight, dry weight, germination (\%), and vigor index of the genotypes stored in different containers at Twelve months after storage.

\begin{tabular}{|c|c|c|c|c|c|c|}
\hline $\begin{array}{l}\text { Genotypes with } \\
\text { treatments }\end{array}$ & $\begin{array}{l}\text { Shoot } \\
\text { length } \\
(\mathrm{cm})\end{array}$ & $\begin{array}{c}\text { Root } \\
\text { length } \\
(\mathrm{cm})\end{array}$ & $\begin{array}{c}\text { Fresh } \\
\text { weight }(g)\end{array}$ & $\begin{array}{c}\text { Dry } \\
\text { weight } \\
\text { (g) }\end{array}$ & $\begin{array}{c}\text { Germination } \\
(\%)\end{array}$ & $\begin{array}{l}\text { Vigor } \\
\text { index }\end{array}$ \\
\hline $\mathrm{T}_{1} \mathrm{G}_{1}$ & 7.22 & 16.34 & 0.11 & 0.07 & 84.67 & 1996.23 \\
\hline $\mathrm{T}_{1} \mathrm{G}_{2}$ & 8.12 & 12.21 & 0.25 & 0.04 & 85.33 & 1732.97 \\
\hline $\mathrm{T}_{1} \mathrm{G}_{3}$ & 7.77 & 12.74 & 0.19 & 0.05 & 86.67 & 1777.80 \\
\hline $\mathrm{T}_{1} \mathrm{G}_{4}$ & 7.03 & 13.11 & 0.15 & 0.05 & 84.67 & 1705.16 \\
\hline $\mathrm{T}_{1} \mathrm{G}_{5}$ & 7.29 & 13.91 & 0.15 & 0.05 & 85.00 & 1801.48 \\
\hline $\mathrm{T}_{1} \mathrm{G}_{6}$ & 7.50 & 10.15 & 0.18 & 0.04 & 84.00 & 1484.61 \\
\hline $\mathrm{T}_{1} \mathrm{G}_{7}$ & 8.29 & 9.35 & 0.29 & 0.05 & 86.33 & 1524.65 \\
\hline $\mathrm{T}_{1} \mathrm{G}_{8}$ & 7.68 & 11.39 & 0.14 & 0.04 & 87.67 & 1671.09 \\
\hline $\mathrm{T}_{1} \mathrm{G}_{9}$ & 9.97 & 15.86 & 0.15 & 0.04 & 87.67 & 2264.74 \\
\hline $\mathrm{T}_{1} \mathrm{G}_{10}$ & 8.74 & 16.74 & 0.33 & 0.05 & 88.33 & 2544.57 \\
\hline $\mathrm{T}_{2} \mathrm{G}_{1}$ & 8.04 & 14.51 & 0.31 & 0.06 & 87.67 & 1973.54 \\
\hline $\mathrm{T}_{2} \mathrm{G}_{2}$ & 5.03 & 8.06 & 0.55 & 0.13 & 86.67 & 1102.81 \\
\hline $\mathrm{T}_{2} \mathrm{G}_{3}$ & 9.14 & 16.74 & 0.20 & 0.05 & 86.33 & 2232.18 \\
\hline $\mathrm{T}_{2} \mathrm{G}_{4}$ & 6.50 & 11.81 & 0.28 & 0.04 & 87.00 & 1591.07 \\
\hline $\mathrm{T}_{2} \mathrm{G}_{5}$ & 8.91 & 16.80 & 0.22 & 0.02 & 86.00 & 2212.82 \\
\hline $\mathrm{T}_{2} \mathrm{G}_{6}$ & 5.07 & 7.87 & 0.20 & 0.03 & 87.33 & 1131.38 \\
\hline $\mathrm{T}_{2} \mathrm{G}_{7}$ & 4.71 & 6.53 & 0.10 & 0.01 & 86.00 & 966.48 \\
\hline $\mathrm{T}_{2} \mathrm{G}_{8}$ & 5.67 & 10.24 & 0.14 & 0.04 & 85.67 & 1362.68 \\
\hline $\mathrm{T}_{2} \mathrm{G}_{9}$ & 11.74 & 16.47 & 0.13 & 0.04 & 89.33 & 2523.29 \\
\hline $\mathrm{T}_{2} \mathrm{G}_{10}$ & 12.34 & 16.78 & 0.18 & 0.07 & 88.00 & 2546.54 \\
\hline $\mathrm{T}_{3} \mathrm{G}_{1}$ & 7.21 & 17.85 & 0.11 & 0.04 & 85.67 & 2144.00 \\
\hline $\mathrm{T}_{3} \mathrm{G}_{2}$ & 6.75 & 10.74 & 0.38 & 0.04 & 85.67 & 1496.04 \\
\hline $\mathrm{T}_{3} \mathrm{G}_{3}$ & 7.58 & 11.21 & 0.12 & 0.07 & 86.00 & 1579.81 \\
\hline $\mathrm{T}_{3} \mathrm{G}_{4}$ & 7.54 & 13.80 & 0.15 & 0.06 & 86.00 & 1857.69 \\
\hline $\mathrm{T}_{3} \mathrm{G}_{5}$ & 6.84 & 13.44 & 0.08 & 0.03 & 86.00 & 1840.56 \\
\hline $\mathrm{T}_{3} \mathrm{G}_{6}$ & 7.41 & 9.41 & 0.26 & 0.02 & 85.67 & 1379.36 \\
\hline $\mathrm{T}_{3} \mathrm{G}_{7}$ & 7.35 & 8.85 & 0.29 & 0.04 & 86.33 & 1454.97 \\
\hline $\mathrm{T}_{3} \mathrm{G}_{8}$ & 8.37 & 10.58 & 0.37 & 0.10 & 86.33 & 1549.41 \\
\hline $\mathrm{T}_{3} \mathrm{G}_{9}$ & 9.21 & 18.41 & 0.19 & 0.03 & 86.33 & 2552.37 \\
\hline $\mathrm{T}_{3} \mathrm{G}_{10}$ & 11.47 & 18.54 & 0.40 & 0.12 & 86.00 & 2580.86 \\
\hline $\mathrm{T}_{4} \mathrm{G}_{1}$ & 9.53 & 10.42 & 0.28 & 0.05 & 84.67 & 1598.23 \\
\hline $\mathrm{T}_{4} \mathrm{G}_{2}$ & 6.99 & 9.26 & 0.14 & 0.04 & 85.33 & 1272.33 \\
\hline $\mathrm{T}_{4} \mathrm{G}_{3}$ & 7.31 & 11.82 & 0.16 & 0.03 & 85.33 & 1105.91 \\
\hline
\end{tabular}




\begin{tabular}{|l|c|c|c|c|c|c|}
\hline $\mathrm{T}_{4} \mathrm{G}_{4}$ & 6.92 & 14.91 & 0.14 & 0.05 & 85.67 & 1869.13 \\
\hline $\mathrm{T}_{4} \mathrm{G}_{5}$ & 6.78 & 9.37 & 0.27 & 0.06 & 85.33 & 1439.84 \\
\hline $\mathrm{T}_{4} \mathrm{G}_{6}$ & 8.35 & 13.48 & 0.17 & 0.05 & 86.67 & 1925.42 \\
\hline $\mathrm{T}_{4} \mathrm{G}_{7}$ & 12.32 & 17.42 & 0.29 & 0.08 & 86.00 & 2558.28 \\
\hline $\mathrm{T}_{4} \mathrm{G}_{8}$ & 7.89 & 11.52 & 0.15 & 0.10 & 86.33 & 1674.87 \\
\hline $\mathrm{T}_{4} \mathrm{G}_{9}$ & 8.93 & 18.51 & 0.15 & 0.06 & 86.33 & 2370.59 \\
\hline $\mathrm{T}_{4} \mathrm{G}_{10}$ & 11.54 & 16.09 & 0.15 & 0.05 & 86.00 & 2376.96 \\
\hline $\mathrm{T}_{5} \mathrm{G}_{1}$ & 7.59 & 16.34 & 0.11 & 0.07 & 84.67 & 2029.51 \\
\hline $\mathrm{T}_{5} \mathrm{G}_{2}$ & 8.14 & 12.21 & 0.25 & 0.04 & 85.00 & 1728.35 \\
\hline $\mathrm{T}_{5} \mathrm{G}_{3}$ & 7.84 & 12.74 & 0.19 & 0.05 & 86.33 & 1789.79 \\
\hline $\mathrm{T}_{5} \mathrm{G}_{4}$ & 7.03 & 13.11 & 0.12 & 0.05 & 84.67 & 1705.25 \\
\hline $\mathrm{T}_{5} \mathrm{G}_{5}$ & 7.36 & 13.91 & 0.15 & 0.05 & 85.00 & 1807.50 \\
\hline $\mathrm{T}_{5} \mathrm{G}_{6}$ & 7.50 & 10.13 & 0.18 & 0.04 & 84.33 & 1489.41 \\
\hline $\mathrm{T}_{5} \mathrm{G}_{7}$ & 8.29 & 9.38 & 0.29 & 0.05 & 86.00 & 1518.10 \\
\hline $\mathrm{T}_{5} \mathrm{G}_{8}$ & 7.91 & 11.39 & 0.14 & 0.04 & 85.67 & 1653.39 \\
\hline $\mathrm{T}_{5} \mathrm{G}_{9}$ & 10.18 & 15.86 & 0.15 & 0.04 & 87.00 & 2268.25 \\
\hline $\mathrm{T}_{5} \mathrm{G}_{10}$ & 12.40 & 16.74 & 0.33 & 0.08 & 86.00 & 2507.26 \\
\hline $\mathbf{S E m}_{1}(\mathbf{m})$ & $\mathbf{0 . 1 6 7}$ & $\mathbf{0 . 1 2 2}$ & $\mathbf{0 . 0 1 1}$ & $\mathbf{0 . 0 0 8}$ & $\mathbf{0 . 0 5 3}$ & $\mathbf{1 7 . 3 6}$ \\
\hline $\mathbf{L S D}(\mathbf{0 . 0 5})$ & $\mathbf{0 . 4 9 2}$ & $\mathbf{0 . 3 7 6}$ & $\mathbf{0 . 0 3 4}$ & $\mathbf{0 . 0 2 1}$ & $\mathbf{0 . 1 4 9}$ & $\mathbf{5 2 . 1 4}$ \\
\hline
\end{tabular}

NB: Kailash- $\mathrm{G}_{1}$, Gobindabhog- $\mathrm{G}_{2}$, Kalma- $\mathrm{G}_{3}$, Dudheswar- $\mathrm{G}_{4}$, Malabati- $\mathrm{G}_{5}$, Kanakchur- $\mathrm{G}_{6}$, Kalojira- $\mathrm{G}_{7}$, Radhatilak- $\mathrm{G}_{8}$, IR36- G9 and Pusa-1509- $\mathrm{G}_{10}$. Earthen pot with seed moisture content $10 \%-\mathrm{T}_{1}$, cloth bag with seed moisture content $10 \%-\mathrm{T}_{2}$, aluminum foil packet with seed moisture content $10 \%-\mathrm{T}_{3}$, aluminum foil packet with seed moisture content $8 \%$ - $\mathrm{T}_{4}$, and polythene packet with seed moisture content $10 \%-\mathrm{T}_{5}$.

At Twelve months of storage, seedling root length was recorded as highest when seeds were stored in $\mathrm{T}_{3} \mathrm{G}_{10}(18.54 \mathrm{~cm})$ and it was lowest when stored for $\mathrm{T}_{2} \mathrm{G}_{7}(6.53 \mathrm{~cm})$. Among the ten genotypes, the average shoot length was highest in $\mathrm{T}_{5} \mathrm{G}_{10}(12.40 \mathrm{~cm})$ and lowest for $\mathrm{T}_{2} \mathrm{G}_{7}$ $(4.71 \mathrm{~cm})$. In the case of fresh weight showed highest for $\mathrm{T}_{2} \mathrm{G}_{2}(0.55 \mathrm{~g})$ and lowest for $\mathrm{T}_{2} \mathrm{G}_{2}$ $(0.13 \mathrm{~g})$. The highest dry weight was recorded for $\mathrm{T}_{4} \mathrm{G}_{5}(0.36 \mathrm{~g})$ followed by $\mathrm{T}_{2} \mathrm{G}_{4}, \mathrm{~T}_{2} \mathrm{G}_{6}$, and $\mathrm{T}_{2} \mathrm{G}_{5}$. Among the ten genotypes germination percentage was highest in $\mathrm{T}_{2} \mathrm{G}_{9},(89.33)$ and lowest in $\mathrm{T}_{1} \mathrm{G}_{6}$ (84.00). Vigor index recorded highest in $\mathrm{T}_{3} \mathrm{G}_{10}$ (2580.86) and lowest in $\mathrm{T}_{2} \mathrm{G}_{7}$ (966.48).

So, it can be concluded that $\mathrm{T}_{2} \mathrm{G}_{9}$ (IR-36 store in a cloth bag with seed moisture content $10 \%$ ) recorded the highest germination percentage followed by $\mathrm{T}_{1} \mathrm{G}_{8}$, and $\mathrm{T}_{4} \mathrm{G}_{6}$ at Twelve months after storage while $\mathrm{T}_{3} \mathrm{G}_{10}$ i.e., Pusa- 1509 stored in aluminum foil packet recorded highest vigor index followed by $\mathrm{T}_{4} \mathrm{G}_{7}$ and $\mathrm{T}_{3} \mathrm{G}_{9}$.

Table 9. Mean value with standard deviation for seedling shoot length, root length, fresh weight, dry weight, germination (\%), and vigor index of seed stored in different containers after twelve months of storage.

\begin{tabular}{|l|l|l|l|l|l|l|l|}
\hline Treatments & $\begin{array}{c}\text { No } \\
\text { of } \\
\text { Geno }\end{array}$ & $\begin{array}{c}\text { Shoot } \\
\text { length } \\
(\mathrm{cm}) \\
\text { Mean } \pm \mathrm{SD}\end{array}$ & $\begin{array}{c}\text { Root length } \\
(\mathrm{cm}) \\
\text { Mean } \pm \mathrm{SD}\end{array}$ & $\begin{array}{c}\text { Fresh weight } \\
(\mathrm{g}) \text { Mean } \pm \mathrm{SD}\end{array}$ & $\begin{array}{c}\text { Dry weight } \\
(\mathrm{g}) \text { Mean } \pm \mathrm{SD}\end{array}$ & $\begin{array}{c}\text { Germination\% } \\
\text { Mean } \pm \mathrm{SD}\end{array}$ & GI Mean $\pm \mathrm{SD}$ \\
\hline $\mathrm{T}_{1}$ & 10 & $\begin{array}{l}7.96^{\mathrm{A}} \\
\pm 0.88\end{array}$ & $13.18^{\mathrm{A}} \pm 2.55$ & $0.224^{\mathrm{A}} \pm 0.143$ & $0.048^{\mathrm{A}} \pm 0.009$ & $86.03^{\mathrm{A}} \pm 1.51$ & $1850.33^{\mathrm{A}} \pm 331.56$ \\
\hline
\end{tabular}




\begin{tabular}{|l|l|l|c|c|c|c|c|}
\hline $\mathrm{T}_{2}$ & 10 & $\begin{array}{l}7.71^{\mathrm{A}} \\
\pm 2.78\end{array}$ & $12.95^{\mathrm{A}} \pm 2.98$ & $0.231^{\mathrm{A}} \pm 0.129$ & $0.077^{\mathrm{A}} \pm 0.044$ & $87.0^{\mathrm{A}} \pm 1.21$ & $1764.27^{\mathrm{A}} \pm 607.16$ \\
\hline $\mathrm{T}_{3}$ & 10 & $7.97^{\mathrm{A}} \pm 1.42$ & $13.28^{\mathrm{A}} \pm 3.76$ & $0.335^{\mathrm{A}} \pm 0.283$ & $0.055^{\mathrm{A}} \pm 0.32$ & $86.0^{\mathrm{B}} \pm 0.26$ & $1996.96^{\mathrm{A}} \pm 827.47$ \\
\hline $\mathrm{T}_{4}$ & 10 & $8.65^{\mathrm{A}} \pm 1.95$ & $13.28^{\mathrm{A}} \pm 3.33$ & $0.243^{\mathrm{A}} \pm 0.203$ & $0.087^{\mathrm{A}} \pm 0.097$ & $85.76^{\mathrm{B}} \pm 0.60$ & $2306.94^{\mathrm{A}} \pm 1360.24$ \\
\hline $\mathrm{T}_{5}$ & 10 & $8.42^{\mathrm{A}} \pm 1.64$ & $13.18^{\mathrm{A}} \pm 2.54$ & $0.221^{\mathrm{A}} \pm 0.145$ & $0.051^{\mathrm{A}} \pm 0.013$ & $85.46^{\mathrm{B}} \pm 0.86$ & $2059.67^{\mathrm{A}} \pm 692.92$ \\
\hline SEm( \pm$)$ & & $\mathbf{0 . 2 7 5}$ & $\mathbf{0 . 5 1 3}$ & $\mathbf{0 . 0 2 8}$ & $\mathbf{8 . 1 6}$ & $\mathbf{0 . 1 4 4}$ & $\mathbf{1 2 4 . 6 0}$ \\
\hline LSD (0.05) & $\mathbf{N S}$ & $\mathbf{N S}$ & $\mathbf{N S}$ & $\mathbf{N S}$ & $\mathbf{0 . 4 2 5 9} *$ & $\mathbf{N S}$ \\
\hline
\end{tabular}

NB: Earthen pot with seed moisture content $10 \%$ - $\mathrm{T}_{1}$, cloth bag with seed moisture content $10 \%-\mathrm{T}_{2}$, aluminum foil packet- $\mathrm{T}_{3}$, aluminum foil packet with seed moisture content $8 \%-\mathrm{T}_{4}$, and polythene packet with seed moisture content $10 \%-\mathrm{T}_{5}$.

Twelve months after storage, treatments over genotypes highest mean value of shoot length observed in $\mathrm{T}_{2}(8.65 \mathrm{~cm})$ and the lowest in $\mathrm{T}_{4}(7.71)$. The deviation from the mean value was ranged from 0.88 to 2.78 and the $\mathrm{T}_{2}$ showed the highest. All the genotypes showed nonsignificant variation they were grouped under same group i.e. under A. In case of root length longest was recorded in $\mathrm{T}_{3}$ and $\mathrm{T}_{4}(13.28)$ and shortest in $\mathrm{T}_{2}(12.95 \mathrm{~cm})$. The deviation from the mean value was ranged from 2.54 to 3.76 and the $T_{3}$ showed the highest. All genotypes showed non-significant variation they were grouped under the same group i.e. under A. The highest fresh weight was observed in $\mathrm{T}_{3}(0.335 \mathrm{~g})$ and the lowest in $\mathrm{T}_{5}(0.221 \mathrm{~g})$, the deviation from the mean value was ranges from 0.129 to 0.283 and the $\mathrm{T}_{3}$ was shows highest. All genotypes showed nonsignificant in variation they were grouped under same group i.e. under A. Dry weight was observed highest in $\mathrm{T}_{4}(0.087 \mathrm{~g})$ and lowest in $\mathrm{T}_{1}(0.048 \mathrm{~g})$. The deviation from the mean value was ranged from 0.009 to 0.32 and the $\mathrm{T}_{3}$ was shown the highest. All genotypes showed nonsignificant variation they were grouped under the same group i.e. under A. Highest germination percentage was recorded in $\mathrm{T}_{2}(87.0)$ and lowest in $\mathrm{T}_{5}$ (85.46). The deviation from the mean value was ranged from 0.26 to 1.51 and the $\mathrm{T}_{1}$ was shown the highest. All genotypes showed significance variation they were grouped under various groups $T_{1} \& T_{2}$ were under group $A$ and $\mathrm{T}_{3}, \mathrm{~T}_{4} \& \mathrm{~T}_{5}$ were grouped under group $\mathrm{B}$. Vigor index was highest in $\mathrm{T}_{4}$ (2306.94) and lowest in $\mathrm{T}_{2}$ (1764.27). The deviation from the mean value was ranged from 331.56 to 1360.24 and the $\mathrm{T}_{3}$ showed the highest. All genotypes showed non-significant variation they were grouped under same group i.e. under A.

After twelve months of storage containers, $\mathrm{T}_{2}$ (cloth bag with seed moisture content $10 \%$ ) maintained the highest germination percentage followed by $\mathrm{T}_{3}$ and $\mathrm{T}_{1}$ and while highest vigor index was determined for $\mathrm{T}_{4}$ (aluminum foil packet with seed moisture content $8 \%$ ) followed by $\mathrm{T}_{5}$ and $\mathrm{T}_{3}$. Aluminum foil packet with seed moisture content $8 \%\left(\mathrm{~T}_{4}\right)$, therefore, is regarded as the best storage material when seeds of this crop, in general, are stored for twelve months.

\section{CONCLUSION}

It can be concluded that aluminum foil packet with seed moisture content of $8 \%$ is the best storage container of rice among the other containers like earthen pot with seed moisture content of $10 \%$, aluminum foil, cloth bag with seed moisture content $10 \%$, and polythene packet with seed moisture content $10 \%$ as every three months interval up to one year, vigor index was observed highest in an aluminum foil packet with seed moisture content of $8 \%$. 


\section{REFERENCES}

Abdul Baki, A. A., \& Anderson, J. D. (1973). Vigor determination in soybean seed by multiple criteria. Crop Science. 13, 630-633.

Biswas, S., Bordolui, S. K. \& Chattopadhyay, P. (2020). Determination of Sowing Time of Parental Lines of Hybrid Rice Seed Production in Microclimatic Condition in Target Regions of West Bengal. International J. of Microbiology Research, 12(5), 1836-1840

Bordolui, S.K., Chattapadhyay, P., \& Chandra, P (2006). Morphological characters of some conserve folk rice genotypes. Environment and Ecology, 24 (2), 421-417.

Bordolui, S.K., Chattopadhyay, P., \& Basu, A. K. (2018). Evaluation of some small seeded aromatic indigenous genotypes for commercial utilization as high value rice. International Journal of Minor Fruits, Medicinal and Aromatic Plants, 4 (1), 40-43.

Bordolui, S.K., Sadhukhan, R., \& Chattopadhyay, P. (2015). Participatory evaluation of some folk rice genotypes. Journal Crop and Weed, 11(2), 59-62.

Chandra, P, Samui, R.C., \& Bordolui, S. K. (2006). Growth, yield attributes and yield of different cultivars of groundnut as affected by potassium application. Journal of crop and weed, 2(1), 37-39.

Ramanadane, T., Ponnuswamy, A. S., \& Raja, K. (2005). Influence of production environment on seed quality. Madras Agricultural Journal, 92(4-6), 200-207.

Roberts, E. H. (1972). Loss of viability and crop yields. Viability of seeds. Chapman and Hall, London, 307-320.

\section{Copyrights}

Copyright for this article is retained by the author(s), with first publication rights granted to the journal. This is an open-access article distributed under the terms and conditions of the Creative Commons Attribution license (http://creativecommons.org/licenses/by/4.0/). 